\title{
Heart Block Caused by Ticagrelor Use in a Patient Who Underwent Adenosine Diastolic Fractional Reserve Assessment: A Case Report
}

\author{
Asseel Al-Bayati ${ }^{\mathrm{a}}$, Kyle Wiseman ${ }^{\mathrm{a}}$, Ndausung Udongwo ${ }^{\mathrm{a}, \mathrm{c}}$, Firas Ajam ${ }^{\mathrm{b}}$, \\ Riple Hansalia ${ }^{\mathrm{b}}$, Renato Apolito ${ }^{\mathrm{b}}$
}

\begin{abstract}
Ticagrelor is a direct and rapid-acting antagonist of the P2Y12-adenosine diphosphate receptor found on platelets. The drug is recommended as a first-line antiplatelet agent in patients with acute coronary syndromes, as evidenced in its superiority compared to clopidogrel according to the Platelet Inhibition and Patient Outcomes study. Specifically, the mechanism of action has been proven to show higher inhibition and less variability in its action on P2Y12 receptors compared to clopidogrel. Additionally, ticagrelor inhibits the equilibrative nucleoside transporter 1 adenosine transporter protein leading to an increased concentration of adenosine in the blood, particularly at sites of ischemia. This effect increases the biological efficacy of ticagrelor in terms of cardioprotection, anticoagulation effects, and anti-inflammatory effects. However, the effects are also thought to be responsible for some of the adverse pharmacological effects reported with ticagrelor, such as bradycardia and ventricular pauses $>3$ seconds. Herein, we report a case of recurrent sinus arrest and ventricular asystole in a patient pre-treated with ticagrelor and subsequent physiological assessment of a coronary lesion with fractional flow reserve using intravenous adenosine infusion.
\end{abstract}

Keywords: Heart block; Sinus arrest; Asystole; Bradycardia; Acute coronary disease; Ticagrelor-induced bradyarrhythmia; Chest pain; Cardiac catheterization

\section{Introduction}

Ticagrelor is commonly used as a first-line antiplatelet agent

Manuscript submitted October 22, 2021, accepted November 18, 2021

Published online December 2, 2021

${ }^{a}$ Department of Internal Medicine, Jersey Shore University Medical Center, Neptune, NJ, USA

bDepartment of Cardiology, Jersey Shore University Medical Center, Neptune, NJ, USA

${ }^{\mathrm{c}}$ Corresponding Author: Ndausung Udongwo, Department of Internal Medicine, Jersey Shore University Medical Center, Neptune, NJ, USA.

Email: ndausung.udongwo@hmhn.org

doi: https://doi.org/10.14740/jmc3815 in the management of ischemic heart disease and acute coronary syndrome (ACS) due to its rapid onset and proven cardiovascular benefits [1]. Though the benefits have been shown to reduce mortality [1], the mechanism of the drug's effect on extracellular adenosine levels can unveil or induce bradyarrhythmias. This may be through a sinoatrial nodal or an atrioventricular mechanism [2, 3]. Many of the bradyarrhythmias resolve after discontinuation of ticagrelor or spontaneously [2]. We present a patient whose conduction abnormality failed to recover despite cessation of ticagrelor, requiring the need for pacemaker placement.

\section{Case Report}

\section{Investigations}

A 64-year-old woman presented with retrosternal chest pain radiating to the jaw and back, which woke her up from sleep. The pain was partially relieved with sublingual nitroglycerin. She has a history of coronary artery disease with stable, $70 \%$ mid-left anterior descending coronary artery and diagonal stenosis lesions that were diagnosed upon pre-op evaluation for a nephrectomy due to left renal cell carcinoma 2 years prior. Her past medical history was also remarkable for hypertension and chronic kidney disease stage 3 .

\section{Diagnosis}

An electrocardiogram (EKG) showed normal sinus rhythm with sinus arrhythmia at a rate of $70 \mathrm{bpm}$ with signs of left ventricular hypertrophy with occasional premature ventricular contractions. There were no ST-segment or T-wave changes and there was normal conduction. Multiple serial troponins on admission and $6 \mathrm{~h}$ apart remained below $0.04 \mathrm{ng} /$ $\mathrm{mL}$. Computed tomography (CT) of the chest and abdomen with contrast angiography revealed a stable aortic aneurysm of $4.5 \mathrm{~cm}$ diameter with no signs of dissection. D-dimer was negative. Brain natriuretic peptide (BNP) was $241 \mathrm{pg} / \mathrm{mL}$. The 2D transthoracic echocardiography showed mild left ventricular hypertrophy with grade 1 diastolic dysfunction with no signs of wall motion abnormalities. During the course of 


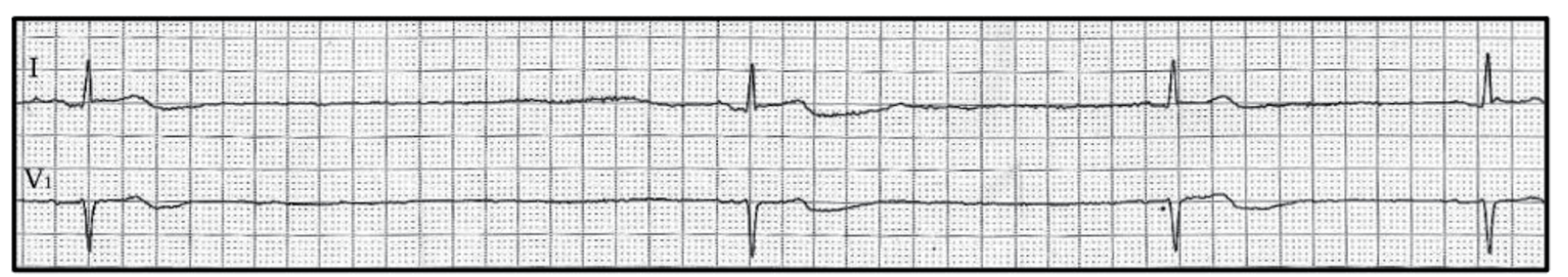

Figure 1. Rhythm strip electrocardiography showing sinus pauses and sinus bradycardia.

her hospitalization, the patient displayed no bradyarrhythmia on continuous telemetry monitoring. The patient was loaded with $324 \mathrm{mg}$ orally (PO) aspirin and started on a nitroglycerin infusion at a rate of $5 \mu \mathrm{g} / \mathrm{min}$, titratable for chest pain control. Due to unresolved chest pain within $48 \mathrm{~h}$ and the high risk for major adverse cardiac events, the patient underwent a diagnostic cardiac catheterization through the standard technique using the right common femoral artery access. The right coronary artery (RCA) was found to be large and dominant with mild disease. The left main coronary artery was patent with no disease. The mid-left anterior descending (LAD) artery showed stable, moderate to severe $70 \%$ stenosis. The left circumflex was angiographically normal. The left ventricular end-diastolic pressure (LVEDP) was $25 \mathrm{~mm} \mathrm{Hg}$ with normal systolic function. Due to no clear signs of ischemia and stable anatomy, the decision was made to manage medically and reevaluate for percutaneous intervention if chest pain persists and is suspected to be of cardiac etiology. Within the next 3 days, the patient continued to have chest pain despite optimized medical management with aspirin $81 \mathrm{mg}$ PO daily, labetalol PO $300 \mathrm{mg}$ twice daily (BID), nitroglycerin infusion, and intravenous (IV) furosemide $40 \mathrm{mg}$ BID. She was again taken to the Cath Lab for a physiological assessment of the coronary artery lesions and possible percutaneous interventions. Immediately preoperatively, the patient was loaded with ticagrelor $180 \mathrm{mg}$ PO. Angiomax anticoagulation was given and a guidewire was introduced into the left coronary artery. The diastolic fractional reserve (DFR) of the first diagonal artery was found to be 0.98 . The proximal LAD could not be accurately interrogated due to severe mid LAD outflow stenosis, so this was treated with a $2.5 \times 12 \mathrm{~mm}$ drug-eluting stent deployed at $14 \mathrm{~atm}$ and further expanded with a $2.75 \times$ $8 \mathrm{~mm}$ non-compliant balloon at $19 \mathrm{~atm}$. DFR of the proximal LAD was 0.95 but it was decided to proceed with fractional flow reserve (FFR) to be certain the lesion was not significant. After calibration and equalization, a Comet Radiwire was maneuvered to the distal LAD and an infusion of IV adenosine at $140 \mu \mathrm{g} / \mathrm{kg} / \mathrm{min}$ was initiated. Within seconds of starting the infusion of adenosine, the patient developed a sinus arrest and asystole for $>8 \mathrm{~s}$, which resolved spontaneously with cessation of adenosine infusion.

\section{Treatment}

The remainder of the procedure was aborted with a plan to continue medical management with aspirin, atorvastatin, labetalol, and ticagrelor. The post-procedure EKG had no changes compared to the baseline EKG, with normal rate and conduction intervals. One hour after the procedure, the patient's telemetry monitor recorded multiple recurrent and symptomatic sinoatrial pauses and asystole for up to $10 \mathrm{~s}$ (Fig. 1). It was suspected immediately that ticagrelor was responsible given the temporal relationship and it was stopped and changed to clopidogrel. Aminophylline $50 \mathrm{mg}$ IV was infused to block the adenosine effect but episodes recurred. It was then decided to place the patient in the cardiac intensive care unit and place a transvenous pacemaker.

\section{Follow-up and outcomes}

The patient remained intermittently dependent on the transvenous pacemaker and received a permanent pacemaker after monitoring over $48 \mathrm{~h}$. She was medically stable and discharged safely to home.

\section{Discussion}

Ticagrelor is a novel direct antiplatelet agent that has shown superiority to the thienopyridine prodrug clopidogrel in preventing cardiovascular death, myocardial infarction, and stroke $(9.8 \%$ vs. $11.7 \%$, a $16 \%$ reduction) among patients with the ACS according to the Platelet Inhibition and Patient Outcomes (PLATO) trial [1]. Furthermore, the PLATO trial demonstrated a significant reduction in the incidence of cardiovascular $(4.0 \%$ vs. $5.1 \%)$ and total $(4.5 \%$ vs. $5.9 \%)$ mortality when comparing ticagrelor and clopidogrel, respectively. This has made it a preferred antiplatelet agent in ACS.

Ticagrelor also has been shown to inhibit the adenosine transporter receptor equilibrative nucleoside transporter (ENT1). The inhibition of ENT1 prevents the intracellular metabolism of adenosine, thereby increasing the half-life and concentration of adenosine in the blood [4]. Ticagrelor's incremental effect on the biological levels of exogenous adenosine might explain the additional cardioprotection, anticoagulation effects, and anti-inflammatory effects. However, this feature may be responsible for some of the reported adverse pharmacological effects as well [5]. In addition to bleeding, "air hunger" dyspnea, nausea, and dizziness, bradyarrhythmia was reported. In a prospective PLATO study of 3,000 patients, Holter monitoring detected ventricular pauses in $6.0 \%$ of patients taking ticagrelor compared to $3.5 \%$ of patients taking 
clopidogrel in the acute phase of the trial (relative risk 1.61, $\mathrm{P}=0.006$ ). These pauses were mainly due to sinoatrial arrest. The phenomenon was noticed mainly during the first week of therapy. At 30 days follow-up, a low incidence of bradyarrhythmia was noted in both study groups with no significant difference. Notably, most sinoatrial pauses were asymptomatic as well [3]. Further studies demonstrated that the reported ventricular pauses were possibly related to a mechanism of ticagrelor independent of its action on ENT1, given that these arrhythmias were sinoatrial in origin and not due to an atrioventricular conduction block [3]. Though adenosine is known to cause an atrioventricular block, it still has some effect on modulating the hyperpolarization-activated current (If), as shown in rabbit myocytes [6]. These findings may still implicate an adenosine-induced sinoatrial dysfunction as a cause of the bradyarrhythmias through ticagrelor's effect on ENT1. This may have been the cause of the bradyarrhythmias in our patient.

Our patient did not have electrophysiological abnormalities prior to ticagrelor administration. It is not fully understood why ventricular asystole occurred. The sinoatrial arrest and asystole may have been caused by an adenosine-mediated atrioventricular block or through the direct effect of ticagrelor on cardiac automaticity and the cardiac conduction system. Regardless of the etiology, the adenosine infusion during the FFR potentiated the bradyarrhythmic effect in our patient without previous electric conduction abnormalities.

Generally, ticagrelor is well tolerated. The conduction abnormalities induced are often asymptomatic without the need for a permanent pacemaker [1]. The abnormalities are sometimes even transient and resolve with discontinuation of ticagrelor [7]. However, a minority of patients did require pacemaker placement due to persistent sinoatrial disease, like the patient reported here [8]; however, unlike our patient, most of the patients requiring a pacemaker had some underlying conduction abnormality [7].

\section{Learning points}

Ticagrelor is a novel antiplatelet agent with significant mortality and morbidity benefits in patients with cardiovascular disease. However, bradyarrhythmias are possible adverse event. Multiple mechanisms of action with ticagrelor contribute to this phenomenon, including involvement with the adenosine ENT1 receptor. Thus, when performing FFR with adenosine during cardiac catheterization, awareness of this potential ensuing arrhythmia in patients on ticagrelor is vital.

\section{Acknowledgments}

None to declare.

\section{Financial Disclosure}

None to declare.

\section{Conflict of Interest}

None to declare.

\section{Informed Consent}

Informed consent was obtained from the patient before presentation of this manuscript.

\section{Author Contributions}

Each author has been individually involved in and has made substantial contributions to conceptions and designs, acquisition of data, analysis, interpretation of data, drafting and editing the manuscript. Assel Al-Bayati contributed to the designs, acquisition of data and analysis; Kyle Wiseman contributed to the analysis, interpretation of data and editing the manuscript; Ndausung Udongwo contributed to the designs, and editing the manuscript; Firas Ajam contributed to the interpretation of data; Riple Hansalia contributed to the interpretation of data; Renato Apolito contributed to the interpretation of data and editing of manuscript.

\section{Data Availability}

The authors declare that data supporting the findings of this study are available within the article.

\section{References}

1. Wallentin L, Becker RC, Budaj A, Cannon CP, Emanuelsson H, Held C, Horrow J, et al. Ticagrelor versus clopidogrel in patients with acute coronary syndromes. N Engl J Med. 2009;361(11):1045-1057.

2. Waldmann V, Laredo M, Nigam A, Khairy P. Cyclical sinus bradycardia and atrioventricular block induced by ticagrelor. HeartRhythm Case Rep. 2018;4(11):527529.

3. Scirica BM, Cannon CP, Emanuelsson H, Michelson EL, Harrington RA, Husted S, James S, et al. The incidence of bradyarrhythmias and clinical bradyarrhythmic events in patients with acute coronary syndromes treated with ticagrelor or clopidogrel in the PLATO (Platelet Inhibition and Patient Outcomes) trial: results of the continuous electrocardiographic assessment substudy. J Am Coll Cardiol. 2011;57(19):1908-1916.

4. Armstrong D, Summers C, Ewart L, Nylander S, Sidaway JE, van Giezen JJ. Characterization of the adenosine pharmacology of ticagrelor reveals therapeutically relevant inhibition of equilibrative nucleoside transporter 1. J Cardiovasc Pharmacol Ther. 2014;19(2):209-219.

5. Wittfeldt A, Emanuelsson H, Brandrup-Wognsen G, van Giezen JJ, Jonasson J, Nylander S, Gan LM. Ticagrelor enhances adenosine-induced coronary vasodilatory re- 
sponses in humans. J Am Coll Cardiol. 2013;61(7):723727.

6. Zaza A, Rocchetti M, DiFrancesco D. Modulation of the hyperpolarization-activated current (I(f)) by adenosine in rabbit sinoatrial myocytes. Circulation. 1996;94(4):734741.
7. De Maria E, Borghi A, Modonesi L, Cappelli S. Ticagrelor therapy and atrioventricular block: Do we need to worry? World J Clin Cases. 2017;5(5):178-182.

8. Goldberg A, Rosenfeld I, Nordkin I, Halabi M. Lifethreatening complete atrioventricular block associated with ticagrelor therapy. Int J Cardiol. 2015;182:379-380. 